PROCEEDINGS OF THE

AMERICAN MATHEMATICAL SOCIETY

Volume 124, Number 2, February 1996

\title{
CONTINUITY OF THE COMPLEX MONGE-AMPÈRE OPERATOR
}

\author{
YANG XING
}

(Communicated by Eric Bedford)

\begin{abstract}
The complex Monge-Ampère operator $\left(d d^{c}\right)^{n}$ is an important tool in complex analysis. It would be interesting to find the right notion of convergence $u_{j} \rightarrow u$ such that $\left(d d^{c} u_{j}\right)^{n} \rightarrow\left(d d^{c} u\right)^{n}$ in the weak topology. In this paper, using the $C_{n-1}$-capacity, we give a sufficient condition of the weak convergence $\left(d d^{c} u_{j}\right)^{n} \rightarrow\left(d d^{c} u\right)^{n}$. We also show that our condition is quite sharp in some case.
\end{abstract}

\section{INTRODUCTION}

Let $\Omega$ be an open subset in $\mathbb{C}^{n}$. PSH $(\Omega)$ will stand for the set of all plurisubharmonic (psh) functions on $\Omega$. We use the standard notations $d=\partial+\bar{\partial}$ and $d^{c}=i(\bar{\partial}-\partial)$. The complex Monge-Ampère operator $\left(d d^{c}\right)^{n}$ is, via integrations by parts, well defined on $P S H(\Omega) \cap L_{l o c}^{\infty}(\Omega)$ and is continuous under monotone limits, that is, $\left(d d^{c} u_{j}\right)^{n} \rightarrow\left(d d^{c} u\right)^{n}$ in the sense of currents if the monotone sequence of functions $u_{j}$ converges to $u$ almost everywhere in $\Omega$, see [B-T2]. This basic fact implies an important property that all psh functions are quasi-continuous with respect to the capacity $C_{n}$ defined by

$$
C_{n}(E)=C_{n}(E, \Omega)=\sup \left\{\int_{E}\left(d d^{c} u\right)^{n} ; u \in P S H(\Omega), 0<u<1\right\}
$$

for any Borel set $E \subset \Omega$, see [B-T2].

A natural question is to find the right notion of convergence $u_{j} \rightarrow u$ such that $\left(d d^{c} u_{j}\right)^{n} \rightarrow\left(d d^{c} u\right)^{n}$ in the sense of currents. Cegrell [C] and Lelong [L2] have observed that the convergence of $u_{j}$ to $u$ in $L_{l o c}^{1}$ is not enough. In the papers [R] and $[\mathrm{X}]$, the Hausdorff content, an outer measure close to Hausdorff measure, was used to obtain a sufficient condition of the weak convergence $\left(d d^{c} u_{j}\right)^{n} \rightarrow\left(d d^{c} u\right)^{n}$. In section 1 of this paper, by slightly modifying the capacity $C_{n}$, we give a weaker condition to ensure $\left(d d^{c} u_{j}\right)^{n} \rightarrow\left(d d^{c} u\right)^{n}$. To see the sharpness of our conditions, we shall discuss properties of convergence of functions $u_{j}$ to $u$ if the corresponding Monge-Ampère measures converge in some sense. Finally, in section 2 we give an application of our results to the range of the Monge-Ampère operator.

The author would like to thank Urban Cegrell for helpful comments on an earlier version of this paper.

Received by the editors August 15, 1994.

1991 Mathematics Subject Classification. Primary 32F07; Secondary 32F05.

Key words and phrases. Plurisubharmonic function, complex Monge-Ampère operator.

Partially supported by the Swedish Natural Science Research Council. 


\section{Continuity of the operator $\left(d d^{c}\right)^{n}$}

Similar to the paper [B-T2], we introduce an inner capacity $C_{n-1}$ by

$$
C_{n-1}(E)=C_{n-1}(E, \Omega)=\sup \left\{C_{n-1}(K) ; K \text { is a compact subset of } E\right\}
$$

for each subset $E$ of $\Omega$, where for the compact subset $K$ we set

$$
C_{n-1}(K)=\sup \left\{\int_{K}\left(d d^{c} u\right)^{n-1} \wedge d d^{c}|z|^{2} ; u \in P S H(\Omega), 0<u<1\right\} .
$$

By the expansion of $\left(d d^{c}\left(u+|z|^{2}\right)\right)^{n}$, we see that there exists a constant $A_{\Omega}>$ 0 such that $C_{n-1}(E) \leq A_{\Omega} C_{n}(E)$ for all subsets $E$ in $\Omega$. On the other hand, using the Chern-Levine-Nirenberg estimate, see [B-T2], and Lelong's result that $r^{-2} \int_{\left\{\left|z-z_{0}\right|<r\right\}}\left(d d^{c} u\right)^{n-1} \wedge d d^{c}|z|^{2}$ for each $u \in P S H(\Omega)$ and each $z_{0}$ in $\Omega$ is an increasing function of $r$, see [L1], we can easily prove that for every $\Omega_{1} \subset \subset \Omega$ there exists a constant $A_{\Omega_{1}}>0$ such that $C_{n-1}(E) \leq A_{\Omega_{1}} H_{2}(E)$ holds for any subset $E$ of $\Omega_{1}$, where $H_{2}$ denotes Hausdorff 2-measure. This implies that $C_{n}$-capacity cannot be estimated by $C_{n-1}$-capacity, since there exists a compact subset $E$ in $\mathbb{C}^{n}$ with the Hausdorff dimension strictly less than 2 and with the positive $C_{n}$-capacity (or equivalently, $E$ is not a pluripolar set).

Recall that a sequence of functions $u_{j}$ is said to converge to a function $u$ in $C_{l}$-capacity on a set $E$, where $l=n-1$ or $n$, if for each constant $\delta>0$ we have

$$
C_{l}\left\{z \in E ;\left|u_{j}(z)-u(z)\right|>\delta\right\} \longrightarrow 0, \quad \text { as } \quad j \rightarrow \infty .
$$

Using the quasi-continuity of psh functions with respect to $C_{n}$-capacity, see [B-T2], we have the following theorem.

Theorem 1. Suppose that $u_{j}$ are locally uniformly bounded psh functions in $\Omega$ and suppose that $u \in P S H(\Omega) \cap L_{\text {loc }}^{\infty}(\Omega)$. Then the following assertions hold.

(i) If $u_{j} \rightarrow u$ in $C_{n-1}$-capacity on each $E \subset \subset \Omega$, then $\left(d d^{c} u_{j}\right)^{n} \rightarrow\left(d d^{c} u\right)^{n}$ in the sense of currents.

(ii) If $u_{j} \rightarrow u$ in $C_{n}$-capacity on each $E \subset \subset \Omega$, then $u_{j}\left(d d^{c} u_{j}\right)^{n} \rightarrow u\left(d d^{c} u\right)^{n}$ in the sense of currents.

Proof. We only give the proof of assertion (i) which in fact also serves as the proof of assertion (ii). We shall show by induction that for each positive integer $k \leq n$, $\left(d d^{c} u_{j}\right)^{k} \rightarrow\left(d d^{c} u\right)^{k}$. It is clear for $k=1$ since the convergence assumption implies that $u_{j} \rightarrow u$ in $L_{l o c}^{1}(\Omega)$. Assume that it is true for $k=q<n$. We prove now that $u_{j}\left(d d^{c} u_{j}\right)^{q} \rightarrow u\left(d d^{c} u\right)^{q}$, which implies that the statement is true for $k=q+1$. For a given $\varepsilon>0$ we let $u=\phi+\psi$ on $\Omega$ where $\phi$ is continuous, $\psi=0$ outside a subset of $\Omega$ with the $C_{n}$-capacity $<\varepsilon$, and the supremum norm of $\psi$ depends only on the function $u$, see [B-T2]. Write

$$
\begin{aligned}
& u_{j}\left(d d^{c} u_{j}\right)^{q}-u\left(d d^{c} u\right)^{q} \\
& \quad=\left(u_{j}-u\right)\left(d d^{c} u_{j}\right)^{q}+\psi\left[\left(d d^{c} u_{j}\right)^{q}-\left(d d^{c} u\right)^{q}\right]+\phi\left[\left(d d^{c} u_{j}\right)^{q}-\left(d d^{c} u\right)^{q}\right] .
\end{aligned}
$$

The inductive assumption gives that the last term on the right-hand side converges to 0 in the sense of currents. On the other hand, since we have the positive measures 
$\left(d d^{c} u_{j}\right)^{q} \wedge\left(d d^{c}|z|^{2}\right)^{n-q} \leq\left(d d^{c}\left(u_{j}+|z|^{2}\right)\right)^{n-1} \wedge d d^{c}|z|^{2}$ which are majorized by $C_{n-1}$-capacity multiplied by a constant not depending on $j$, it follows from the convergence assumption that the first term on the right-hand side also converges to 0 in the sense of currents. Similarly, we can get that the second term makes arbitrarily small mass for all $j$ by choosing $\varepsilon$ small enough. Therefore we have obtained the weak convergence $u_{j}\left(d d^{c} u_{j}\right)^{q} \rightarrow u\left(d d^{c} u\right)^{q}$, and the proof is complete.

Combined with Dini's theorem, Theorem 1 implies that the Monge-Ampère operator $\left(d d^{c}\right)^{n}$ is continuous under monotone limits. However, Theorem 1 is quite sharp as the following result shows.

Theorem 2. Let $E \subset \subset \Omega \subset \mathbb{C}^{n}$. Suppose that $u_{j}$ are locally uniformly bounded psh functions in $\Omega$ and suppose that there exists a function $u \in P S H(\Omega) \cap L_{l o c}^{\infty}(\Omega)$ such that $u_{j}=u$ on $\Omega \backslash E$ for all $j$. Then the following assertions hold.

(i) $u_{j} \rightarrow u$ in $C_{n}$-capacity on $\Omega$ if and only if $u_{j}\left(d d^{c} u_{j}\right)^{n}, u\left(d d^{c} u_{j}\right)^{n}$ and $u_{j}\left(d d^{c} u\right)^{n}$ converge to $u\left(d d^{c} u\right)^{n}$ in the sense of currents.

(ii) $u_{j} \rightarrow u$ in $C_{n-1}$-capacity on $\Omega$ if and only if $\left(d d^{c} u_{j}\right)^{n},\left(d d^{c} u_{j}\right)^{n-1} \wedge d d^{c} u$ and $\left(d d^{c} u\right)^{n-1} \wedge d d^{c} u_{j}$ converge to $\left(d d^{c} u\right)^{n}$ in the sense of currents.

(iii) In the special case $n=2$, we have that $u_{j} \rightarrow u$ in $C_{1}$-capacity on $\Omega$ if and only if $\left(d d^{c} u_{j}\right)^{2} \rightarrow\left(d d^{c} u\right)^{2}$ in the sense of currents and $u_{j} \rightarrow u$ in $L_{l o c}^{1}(\Omega)$.

(iv) Under the additional assumption that either $u_{j} \geq u$ or $u_{j} \leq u$ holds in $\Omega$ for each $j$, we have that $u_{j} \rightarrow u$ in $C_{n-1}$-capacity on $\Omega$ if and only if $\left(d d^{c} u_{j}\right)^{n} \rightarrow$ $\left(d d^{c} u\right)^{n}$ in the sense of currents.

Proof. All of the "only if" parts follow from the proof of Theorem 1. We only need to show the "if" parts. Assume that $\delta$ is a positive constant and assume that $w$ is a function in $\operatorname{PSH}(\Omega)$ with $0<w<1$. Choose an open set $\Omega^{\prime}$ such that $E \subset \subset \Omega^{\prime} \subset \subset \Omega$.

(i) Using integration by parts and the Schwarz inequality, we have

$$
\begin{gathered}
\int_{\left\{\left|u_{j}-u\right|>\delta\right\}}\left(d d^{c} w\right)^{n} \leq \frac{1}{\delta^{2}} \int_{\Omega^{\prime}}\left(u_{j}-u\right)^{2}\left(d d^{c} w\right)^{n}=-\frac{1}{\delta^{2}} \int_{\Omega^{\prime}} d\left(u_{j}-u\right)^{2} \wedge d^{c} w \wedge\left(d d^{c} w\right)^{n-1} \\
\leq A_{1}\left(\int_{\Omega^{\prime}} d\left(u_{j}-u\right)^{2} \wedge d^{c}\left(u_{j}-u\right)^{2} \wedge\left(d d^{c} w\right)^{n-1}\right)^{1 / 2} \\
\leq 2 A_{1} A_{2}\left(\int_{\Omega^{\prime}} d\left(u_{j}-u\right) \wedge d^{c}\left(u_{j}-u\right) \wedge\left(d d^{c} w\right)^{n-1}\right)^{1 / 2}
\end{gathered}
$$

where the constant $A_{1}=1 / \delta^{2}\left(\int_{\Omega^{\prime}} d w \wedge d^{c} w \wedge\left(d d^{c} w\right)^{n-1}\right)^{1 / 2}$ is uniformly bounded for all functions $w \in P S H(\Omega)$ with $0<w<1$, see the Chern-Levine-Nirenberg estimate in [B-T2], and the constant $A_{2}$ exceeds $\left|u_{j}(z)-u(z)\right|$ for all $j$ and $z \in \Omega$. Again by integration by parts, the last integral in the above inequality is equal to

$$
\int_{\Omega^{\prime}} d\left(u_{j}-u\right) \wedge d^{c} w \wedge d d^{c} u_{j} \wedge\left(d d^{c} w\right)^{n-2}-\int_{\Omega^{\prime}} d\left(u_{j}-u\right) \wedge d^{c} w \wedge d d^{c} u \wedge\left(d d^{c} w\right)^{n-2} \text {. }
$$


Applying the Schwarz inequality in each term of this difference, it then turns out from the trivial inequalities $d d^{c} u_{j} \leq d d^{c}\left(u_{j}+u\right)$ and $d d^{c} u \leq d d^{c}\left(u_{j}+u\right)$ that the last difference does not exceed

$$
A_{3}\left(\int_{\Omega^{\prime}} d\left(u_{j}-u\right) \wedge d^{c}\left(u_{j}-u\right) \wedge d d^{c}\left(u_{j}+u\right) \wedge\left(d d^{c} w\right)^{n-2}\right)^{1 / 2},
$$

where the constant $A_{3}$ does not depend on $w$ and $j$ because of the Chern-LevineNirenberg estimate. Now we get an integral involving $\left(d d^{c} w\right)^{n-2}$. (Observe that we began with the integral involving $\left(d d^{c} w\right)^{n-1}$. ) We repeat this argument $n-2$ more times and finally find a constant $A_{4}$, not depending on $w$ and $j$, such that

$$
\begin{gathered}
\int_{\left\{\left|u_{j}-u\right|>\delta\right\}}\left(d d^{c} w\right)^{n} \leq A_{4}\left(\int_{\Omega^{\prime}} d\left(u_{j}-u\right) \wedge d^{c}\left(u_{j}-u\right) \wedge\left(d d^{c}\left(u_{j}+u\right)\right)^{n-1}\right)^{1 / 2^{n}} \\
\leq A_{4}(n !)^{1 / 2^{n-1}}\left(\int_{\Omega^{\prime}} d\left(u_{j}-u\right) \wedge d^{c}\left(u_{j}-u\right) \wedge \sum_{k=0}^{n-1}\left(d d^{c} u_{j}\right)^{n-1-k} \wedge\left(d d^{c} u\right)^{k}\right)^{1 / 2^{n}} \\
=A_{4}(n !)^{1 / 2^{n-1}}\left(\int_{\Omega^{\prime}}\left(u-u_{j}\right)\left(d d^{c} u_{j}-d d^{c} u\right) \wedge \sum_{k=0}^{n-1}\left(d d^{c} u_{j}\right)^{n-1-k} \wedge\left(d d^{c} u\right)^{k}\right)^{1 / 2^{n}} \\
=A_{4}(n !)^{1 / 2^{n-1}}\left(\int_{\Omega^{\prime}}\left(u-u_{j}\right)\left(\left(d d^{c} u_{j}\right)^{n}-\left(d d^{c} u\right)^{n}\right)\right)^{1 / 2^{n}} \cdot
\end{gathered}
$$

Since the function $u-u_{j}$ has a compact support in $\Omega^{\prime}$, it follows from the convergence assumptions that the last integral converges to 0 as $j \rightarrow \infty$. Hence we have proved that for any $\delta>0$

$$
\lim _{j \rightarrow \infty}\left[\sup \left\{\int_{\left\{\left|u_{j}-u\right|>\delta\right\}}\left(d d^{c} w\right)^{n} ; w \in P S H(\Omega), 0<w<1\right\}\right]=0
$$

which completes the proof of assertion (i).

(ii) If we begin with the integral $\int_{\left\{\left|u_{j}-u\right|>\delta\right\}}\left(d d^{c} w\right)^{n-1} \wedge d d^{c}|z|^{2}$ and use the same argument as in the proof of (i), we can get the "if" part of assertion (ii).

(iii) By the quasi-continuity of the function $u$ with respect to $C_{2}$-capacity and the convergence assumption that $u_{j} \rightarrow u$ in $L_{l o c}^{1}(\Omega)$, we can easily get the weak convergence $d d^{c} u_{j} \wedge d d^{c} u \rightarrow\left(d d^{c} u\right)^{2}$. Hence assertion (iii) follows directly from assertion(ii).

(iv) Similar to the proof of (i), we can find a constant $A_{5}$, not depending on $w$ and $j$, such that

$$
\begin{gathered}
\int_{\left\{\left|u_{j}-u\right|>\delta\right\}}\left(d d^{c} w\right)^{n-1} \wedge d d^{c}|z|^{2} \\
\leq A_{5}\left(\int_{\Omega^{\prime}} d\left(u_{j}-u\right) \wedge d^{c}\left(u_{j}-u\right) \wedge\left(d d^{c}\left(u_{j}+u\right)\right)^{n-2} \wedge d d^{c}|z|^{2}\right)^{1 / 2^{n-1}}
\end{gathered}
$$




$$
=A_{5}\left(\int_{\Omega^{\prime}}\left(u-u_{j}\right) d d^{c}\left(u_{j}-u\right) \wedge\left(d d^{c}\left(u_{j}+u\right)\right)^{n-2} \wedge d d^{c}|z|^{2}\right)^{1 / 2^{n-1}} .
$$

Since $u-u_{j}$ does not change sign on $\Omega$ for every $j$, the last integral is majorized by

$$
\begin{aligned}
& \left.(n !)^{2}\left|\int_{\Omega^{\prime}}\left(u_{j}-u\right) d d^{c}\right| z\right|^{2} \wedge \sum_{k=0}^{n-1}\left(d d^{c} u_{j}\right)^{n-1-k} \wedge\left(d d^{c} u\right)^{k} \mid \\
& \quad=\left.(n !)^{2}\left|\int_{\Omega^{\prime}}\right| z\right|^{2}\left(\left(d d^{c} u_{j}\right)^{n}-\left(d d^{c} u\right)^{n}\right) \mid
\end{aligned}
$$

which, by the assumptions, converges to 0 as $j \rightarrow \infty$ and hence assertion (ii) has been proved. We have thus completed the proof of Theorem 2 .

For the "if" parts of Theorem 2, we require that all functions $u_{j}$ coincide with $u$ outside a relatively compact subset of $\Omega$. This requirement cannot be replaced by the weaker restriction that all $u_{j}$ have the same boundary values on $\partial \Omega$ as the function $u$. For instance, psh functions $u_{j}(z)=\max (j \ln |z|,-1)$ converge to the function $u(z) \equiv 0$ nowhere inside the open unit ball in $\mathbb{C}^{n}$ as $j \rightarrow \infty$. However, every function $u_{j}$ for $j>0$ vanishes on the boundary of the unit ball, and its Monge-Ampère measure $\left(d d^{c} u_{j}\right)^{n}$ is a constant multiple of Lebesgue measure on the sphere $|z|=e^{-1 / j}$, which implies that together with the $u$, the functions $u_{j}$ satisfy the other assumptions of the "if" parts of all assertions in Theorem 2, except assertion (iii). In the following we shall give a slightly weaker condition on functions near the boundary instead of that given in Theorem 2. To do this we prefer to set up the following inequality, which we feel has some interest in itself.

Lemma 1. Let $\Omega$ be a bounded open subset in $\mathbb{C}^{n}$ and let $u, v \in P S H(\Omega) \cap L^{\infty}(\Omega)$ satisfy $\liminf _{z \rightarrow \partial \Omega}(u(z)-v(z)) \geq 0$. Then for any constant $r \geq 1$ and all $w_{j} \in$ $\operatorname{PSH}(\Omega)$ with $0 \leq w_{j} \leq 1, j=1,2, \ldots, n$, we have

$$
\begin{aligned}
& \frac{1}{(n !)^{2}} \int_{\{u<v\}}(v-u)^{n} d d^{c} w_{1} \wedge \cdots \wedge d d^{c} w_{n}+\int_{\{u<v\}}\left(r-w_{1}\right)\left(d d^{c} v\right)^{n} \\
& \quad \leq \int_{\{u<v\}}\left(r-w_{1}\right)\left(d d^{c} u\right)^{n} .
\end{aligned}
$$

Proof. We first prove Lemma 1 for continuous functions $u$ and $v$ in $\Omega$. In this case we can assume, without loss of generality, that $\Omega=\{u<v\}$. For each constant $\varepsilon>0$ we define a function $v_{\varepsilon}=\max (u, v-\varepsilon)$, which converges increasingly to $v$ in $\Omega$ as $\varepsilon \searrow 0$ and coincides with $u$ near the boundary $\partial \Omega$. So integrations by parts yield

$$
\begin{aligned}
& \int_{\Omega}\left(v_{\varepsilon}-u\right)^{n} d d^{c} w_{1} \wedge \cdots \wedge d d^{c} w_{n}=\int_{\Omega}\left(w_{n}-1\right) d d^{c}\left(\left(v_{\varepsilon}-u\right)^{n}\right) \wedge d d^{c} w_{1} \wedge \cdots \wedge d d^{c} w_{n-1} \\
& =n(n-1) \int_{\Omega}\left(w_{n}-1\right)\left(v_{\varepsilon}-u\right)^{n-2} d\left(v_{\varepsilon}-u\right) \wedge d^{c}\left(v_{\varepsilon}-u\right) \wedge d d^{c} w_{1} \wedge \cdots \wedge d d^{c} w_{n-1}
\end{aligned}
$$




$$
\begin{aligned}
& +n \int_{\Omega}\left(w_{n}-1\right)\left(v_{\varepsilon}-u\right)^{n-1} d d^{c}\left(v_{\varepsilon}-u\right) \wedge d d^{c} w_{1} \wedge \cdots \wedge d d^{c} w_{n-1} \\
& \quad \leq n \int_{\Omega}\left(v_{\varepsilon}-u\right)^{n-1} d d^{c}\left(v_{\varepsilon}+u\right) \wedge d d^{c} w_{1} \wedge \cdots \wedge d d^{c} w_{n-1} .
\end{aligned}
$$

Repeating this process $n-2$ more times, we have

$$
\begin{gathered}
\int_{\Omega}\left(v_{\varepsilon}-u\right)^{n} d d^{c} w_{1} \wedge \cdots \wedge d d^{c} w_{n} \leq n ! \int_{\Omega}\left(v_{\varepsilon}-u\right)\left(d d^{c}\left(v_{\varepsilon}+u\right)\right)^{n-1} \wedge d d^{c} w_{1} \\
\leq(n !)^{2} \int_{\Omega}\left(v_{\varepsilon}-u\right) d d^{c} w_{1} \wedge \sum_{k=0}^{n-1}\left(d d^{c} v_{\varepsilon}\right)^{n-1-k} \wedge\left(d d^{c} u\right)^{k} \\
=(n !)^{2} \int_{\Omega}\left(r-w_{1}\right)\left(d d^{c} u\right)^{n}-(n !)^{2} \int_{\Omega}\left(r-w_{1}\right)\left(d d^{c} v_{\varepsilon}\right)^{n} .
\end{gathered}
$$

But $\left(r-w_{1}\right)\left(d d^{c} v_{\varepsilon}\right)^{n} \longrightarrow\left(r-w_{1}\right)\left(d d^{c} v\right)^{n}$ as currents when $\varepsilon \searrow 0$, see [B-T2], and hence we have obtained the required inequality for continuous functions $u$ and $v$.

The general case will then follow by an approximation argument. As in the proof of Theorem 4.1 in [B-T2], we may assume that there exists an open $E \subset \subset \Omega$ such that $u(z)-v(z) \geq \delta>0$ for all $z \in \Omega \backslash E$. Otherwise, replace $u$ by $u+2 \delta$ and then let $\delta \searrow 0$. We can thus choose two decreasing sequences of smooth psh functions $u_{k}$ and $v_{j}$ in a neighbourhood $\Omega^{\prime}$ of $\bar{E}$ such that $\lim _{k \rightarrow \infty} u_{k}=u, \lim _{j \rightarrow \infty} v_{j}=v$ in $\Omega^{\prime}$ and $u_{k} \geq v_{j}$ near the boundary $\partial \Omega^{\prime}$. For smooth functions $u_{k}$ and $v_{j}$ in $\Omega^{\prime}$, we have proved the following inequality

$$
\begin{gathered}
\frac{1}{(n !)^{2}} \int_{\Omega^{\prime}} \chi_{\left\{z \in \Omega^{\prime} ; u_{k}<v_{j}\right\}}\left(v_{j}-u_{k}\right)^{n} d d^{c} w_{1} \wedge \cdots \wedge d d^{c} w_{n}+\int_{\left\{z \in \Omega^{\prime} ; u_{k}<v_{j}\right\}}\left(r-w_{1}\right)\left(d d^{c} v_{j}\right)^{n} \\
\leq \int_{\left\{z \in \Omega^{\prime} ; u_{k}<v_{j}\right\}}\left(r-w_{1}\right)\left(d d^{c} u_{k}\right)^{n},
\end{gathered}
$$

where $\chi_{E}$ denotes the characteristic function of a set $E$. Letting $j \rightarrow \infty$ and then $k \rightarrow \infty$ and using Fatou Lemma, we get that the limit inferior of the first term on the left-hand side exceeds

$$
\frac{1}{(n !)^{2}} \int_{\{u<v\}}(v-u)^{n} d d^{c} w_{1} \wedge \cdots \wedge d d^{c} w_{n} .
$$

To handle the other two terms in the same inequality when $j \rightarrow \infty$ and $k \rightarrow \infty$, we first observe that $\left(r-w_{1}\right)\left(d d^{c} u_{k}\right)^{n} \longrightarrow\left(r-w_{1}\right)\left(d d^{c} u\right)^{n}$ and $\left(r-w_{1}\right)\left(d d^{c} v_{j}\right)^{n} \longrightarrow$ $\left(r-w_{1}\right)\left(d d^{c} v\right)^{n}$ as currents, see [B-T2]. Completely repeating the proof of Theorem 4.1 in [B-T2], one can get

$$
\begin{aligned}
& \frac{1}{(n !)^{2}} \int_{\{u<v\}}(v-u)^{n} d d^{c} w_{1} \wedge \cdots \wedge d d^{c} w_{n}+\int_{\{u<v\}}\left(r-w_{1}\right)\left(d d^{c} v\right)^{n} \\
& \quad \leq \int_{\{u \leq v\}}\left(r-w_{1}\right)\left(d d^{c} u\right)^{n} .
\end{aligned}
$$


Finally, applying the last inequality for functions $u+\delta_{1}$ instead of $u$ and letting $\delta_{1} \searrow 0$, we get the required inequality and hence the proof is complete.

Remark. If both sides of the inequality in Lemma 1 are divided by the constant $r$ and then letting $r \rightarrow \infty$, we obtain the inequality

$$
\int_{\{u<v\}}\left(d d^{c} v\right)^{n} \leq \int_{\{u<v\}}\left(d d^{c} u\right)^{n},
$$

which is the result of the comparison theorem for the complex Monge-Ampère operator, due to Bedford and Taylor [B-T2]. Our inequality also implies the following useful estimate.

Lemma 2. Let $\Omega$ be a bounded open set and let $u, v \in P S H(\Omega) \cap L^{\infty}(\Omega)$ satisfy $\limsup _{z \rightarrow \partial \Omega}|u(z)-v(z)|=0$. Then the following inequality

$$
C_{n}\{|u-v| \geq \delta\} \leq \frac{(n !)^{2}}{(1-k)^{n} \delta^{n}}\left\|\left(d d^{c} u\right)^{n}-\left(d d^{c} v\right)^{n}\right\|_{\{|u-v|>k \delta\}}
$$

holds for all constants $\delta>0$ and $0<k<1$, where $\|\mu\|_{E}$ denotes the mass on $E$ of the total variation of a signed measure $\mu$.

Proof. Since $|u-v \pm k \delta| \geq(1-k) \delta$ on the set $\{|u-v| \geq \delta\}$, we deduce from Lemma 1 that for $w_{1}=w_{2}=\cdots=w_{n}=w \in P S H(\Omega)$ with $0<w<1$

$$
\begin{gathered}
\int_{\{|u-v| \geq \delta\}}\left(d d^{c} w\right)^{n} \\
\leq \frac{1}{(1-k)^{n} \delta^{n}}\left[\int_{\{u+\delta \leq v\}}(v-u-k \delta)^{n}\left(d d^{c} w\right)^{n}+\int_{\{v+\delta \leq u\}}(u-v-k \delta)^{n}\left(d d^{c} w\right)^{n}\right] \\
\leq \frac{1}{(1-k)^{n} \delta^{n}}\left[\int_{\{u+k \delta<v\}}(v-u-k \delta)^{n}\left(d d^{c} w\right)^{n}+\int_{\{v+k \delta<u\}}(u-v-k \delta)^{n}\left(d d^{c} w\right)^{n}\right] \\
\leq \frac{(n !)^{2}}{(1-k)^{n} \delta^{n}}\left[\int_{\{|u-v|>k \delta\}}(1-w)\left(\chi_{\{u+k \delta<v\}}-\chi_{\{v+k \delta<u\}}\left(\left(d d^{c} u\right)^{n}-\left(d d^{c} v\right)^{n}\right)\right]\right. \\
\leq \frac{(n !)^{2}}{(1-k)^{n} \delta^{n}}\left\|\left(d d^{c} u\right)^{n}-\left(d d^{c} v\right)^{n}\right\|_{\{|u-v|>k \delta\}},
\end{gathered}
$$

which completes the proof.

As a direct consequence of Lemma 2 we have 
Theorem 3. Suppose that $\Omega$ is a bounded open set and suppose that $u_{j}, u \in$ $P S H(\Omega) \cap L^{\infty}(\Omega)$. If

$$
\begin{aligned}
& \limsup _{z \rightarrow \partial \Omega}\left|u_{j}(z)-u(z)\right|=0 \text { uniformly in } j, \text { and } \\
& \left\|\left(d d^{c} u_{j}\right)^{n}-\left(d d^{c} u\right)^{n}\right\|_{E} \longrightarrow 0 \text { for any subset } E \subset \subset \Omega,
\end{aligned}
$$

then $u_{j} \rightarrow u$ in $C_{n}$-capacity on $\Omega$.

Note that the uniformly vanishing condition (i) of Theorem 3 may be replaced by $\lim \sup _{z \rightarrow \partial \Omega}\left|u_{j}(z)-u(z)\right|=0$ for each $j$, if we assume $\left\|\left(d d^{c} u_{j}\right)^{n}-\left(d d^{c} u\right)^{n}\right\|_{\Omega} \longrightarrow 0$ instead of condition (ii). Otherwise, we cannot weaken condition (i) in such a way, as can be seen from the simple example $u_{j}(z)=\max (j \ln |z|,-1)$ and $u(z) \equiv 0$ in the unit ball. It should also be mentioned that condition (ii) of Theorem 3 cannot be replaced by the weak convergence $\left(d d^{c} u_{j}\right)^{n} \rightarrow\left(d d^{c} u\right)^{n}$ either, as the following example shows.

Example. Assume that $\Omega$ is an open unit ball in $\mathbb{C}^{1}$. By Lemma 2 in $[\mathrm{C}]$ there exists a sequence of subharmonic functions $f_{j}$ with $-1 \leq f_{j} \leq 1 / 2$ in $\Omega$ such that $f_{j}$ converges to a subharmonic function $f$ in the topology of $L_{l o c}^{1}(\Omega)$, but $f_{j} d d^{c} f_{j}$ does not converge to $f d d^{c} f$ as currents. Hence it follows from the monotone convergence theorem in [B-T2] that if the constant $A$ is big enough, $u_{j} d d^{c} u_{j}$ does not converge to $u d d^{c} u$ as currents, where the functions $u_{j}=\max \left(A \ln |z|, f_{j}\right)$ and $u=\max (A \ln |z|, f)$ coincide outside a compact subset of $\Omega$. By Theorem 1 we then have that $u_{j}$ does not converge to $u$ in $C_{1}$-capacity on $\Omega$. However, $u_{j} \rightarrow u$ in $L_{l o c}^{1}(\Omega)$ which gives the weak convergence $d d^{c} u_{j} \rightarrow d d^{c} u$.

In fact, we have an analogue of Theorem 3 for the convergence in $C_{n-1}$-capacity. But we omit the details here, because both the formulation and the proof are completely similar.

\section{RANGe OF THE OPERATOR $\left(d d^{c}\right)^{n}$}

In this section we will give an application of our results to the range of the MongeAmpère operator. We shall study the problem: Find a function $u \in P S H(\Omega) \cap$ $L^{\infty}(\Omega)$ such that $\left(d d^{c} u\right)^{n}=\mu$ on $\Omega$, where $\mu$ is a given positive measure in the bounded open set $\Omega$. One necessary condition of existence of such a solution is that there exists a subsolution for this problem, that is, there exists $v \in P S H(\Omega) \cap L^{\infty}(\Omega)$ such that $\left(d d^{c} v\right)^{n} \geq \mu$. In [C-S] it was shown that for $\mu=f d \lambda$, where $f \in L^{1}(\Omega)$ and $d \lambda$ is the Lebesgue measure, the problem has a solution if there exists a subsolution. Now our result is the following.

Theorem 4. Suppose that there exists a function $v \in P S H(\Omega) \cap L^{\infty}(\Omega)$ such that $\left(d d^{c} v\right)^{n} \geq \mu$ and suppose that there exist Monge-Ampère measures $\mu_{j}=\left(d d^{c} u_{j}\right)^{n}$ in $\Omega$ such that $\left\|\mu_{j}-\mu\right\|_{\Omega} \rightarrow 0$ as $j \rightarrow \infty$, where all functions $u_{j} \in P S H(\Omega) \cap C(\bar{\Omega})$ take the same boundary values on $\partial \Omega$. Then there exists a function $u \in P S H(\Omega) \cap$ $L^{\infty}(\Omega)$ such that $\left(d d^{c} u\right)^{n}=\mu$ in $\Omega$.

Proof. By passing to a subsequence, we may assume that

$$
2^{n}(n !)^{2}\left\|\mu_{j}-\mu\right\|_{\Omega} \leq \frac{1}{2^{(n+2) j}} \quad \text { for } j=1,2, \ldots
$$


So Lemma 2 gives that for any $\delta>0$

$$
\begin{gathered}
C_{n}\left\{\left|u_{j+1}-u_{j}\right| \geq \delta\right\} \leq \frac{2^{n}(n !)^{2}}{\delta^{n}}\left\|\mu_{j+1}-\mu_{j}\right\|_{\Omega} \\
\leq \frac{2^{n}(n !)^{2}}{\delta^{n}}\left(\left\|\mu_{j+1}-\mu\right\|_{\Omega}+\left\|\mu-\mu_{j}\right\|_{\Omega}\right) \leq \frac{1}{\delta^{n} 2^{(n+1) j}} \quad \text { for } j=1,2, \ldots
\end{gathered}
$$

Choose a constant $A$ such that $A \geq|z|$ for all $z \in \Omega$ and choose a constant $c$ such that $c \geq|v(z)|+\left|u_{j}(w)\right|+1$ for all $z \in \Omega, w \in \partial \Omega$ and $j$. From Lemma 1 and the assumption $\left(d d^{c} v\right)^{n} \geq \mu$, it turns out that for each $j$

$$
\begin{aligned}
& \int_{\left\{u_{j}<v-c\right\}}\left(1-\frac{|z|^{2}}{A^{2}}\right) d \mu_{j} \\
& \quad \geq \int_{\left\{u_{j}<v-c\right\}}\left(1-\frac{|z|^{2}}{A^{2}}\right) d \mu+\frac{1}{(n !)^{2} A^{2 n}} \int_{\left\{u_{j}<v-c\right\}}\left(v-c-u_{j}\right)^{n}\left(d d^{c}|z|^{2}\right)^{n} .
\end{aligned}
$$

Let $j \rightarrow \infty$, and since $\left\|\mu_{j}-\mu\right\|_{\Omega} \rightarrow 0$, by Fatou's Lemma we have

$$
\begin{aligned}
& 0 \geq \liminf _{j \rightarrow \infty} \int_{\left\{u_{j}<v-c\right\}}\left(v-c-u_{j}\right)^{n}\left(d d^{c}|z|^{2}\right)^{n} \\
& \geq \int_{\Omega} \liminf _{j \rightarrow \infty}\left(\chi_{\left\{u_{j}<v-c\right\}}\left(v-c-u_{j}\right)^{n}\right)\left(d d^{c}|z|^{2}\right)^{n} \\
& \geq \int_{\Omega} \chi_{\left\{\limsup u_{j}<v-c\right\}}\left(\liminf _{j \rightarrow \infty}\left|v-c-u_{j}\right|\right)^{n}\left(d d^{c}|z|^{2}\right)^{n} \\
& \geq \int_{\left\{\limsup u_{j}<v-c\right\}}\left(v-c-\limsup _{j \rightarrow \infty} u_{j}\right)^{n}\left(d d^{c}|z|^{2}\right)^{n},
\end{aligned}
$$

which implies that $\lim \sup u_{j} \geq v-c$ a.e. in $\Omega$ with respect to the Lebesgue measure, and hence $\lim \sup u_{j}$ is not identically $-\infty$ on any component of $\Omega$. Therefore by Corollary 7.3 in [B-T2] we can find a non-negative psh function $g$ in $\Omega$ such that the set $\left\{g \neq \lim \sup u_{j}\right\}$ is pluripolar; i.e., a set of $C_{n}$-capacity zero. So $g \geq v-c$ a.e. in $\Omega$ with respect to the Lebesgue measure and hence $g$ is a bounded function in $\Omega$. We shall prove that $u_{j} \rightarrow g$ in $C_{n}$-capacity on each compact subset $E$ of $\Omega$. For each $\delta>0$ we have

$$
\begin{gathered}
C_{n}\left(E \cap\left\{\left|g-u_{j}\right| \geq \delta\right\}\right) \leq C_{n}\left(E \cap\left\{\left|g-\sup \left\{u_{j}, u_{j+1}, \ldots\right\}\right| \geq \frac{\delta}{2}\right\}\right) \\
+C_{n}\left\{\left|\sup \left\{u_{j}, u_{j+1}, \ldots\right\}-u_{j}\right| \geq \frac{\delta}{2}\right\} .
\end{gathered}
$$

By Proposition 5.1 in [B-T2] we know that outside a set of $C_{n}$-capacity zero, the functions $\sup \left\{u_{j}, u_{j+1}, \ldots\right\}=\sup ^{*}\left\{u_{j}, u_{j+1}, \ldots\right\}$ decrease to the function $\limsup u_{j}=g$ when $j \nearrow \infty$. Hence combined with the quasi-continuity of psh 
functions, Dini's theorem implies that $\sup \left\{u_{j}, u_{j+1}, \ldots\right\} \rightarrow g$ uniformly on $E$ outside a set of the $C_{n}$-capacity less than any given constant. Thus the first term on the right-hand side of the last inequality converges to 0 as $j \rightarrow \infty$. To see that the second term also converges to 0 as $j \rightarrow \infty$, we first show the following inclusion

$$
\left\{\left|\sup \left\{u_{j}, u_{j+1}, \ldots\right\}-u_{j}\right| \geq \frac{\delta}{2}\right\} \subset \bigcup_{l=0}^{\infty}\left\{\left|u_{l+j+1}-u_{l+j}\right| \geq \frac{\delta}{2^{l+j+2}}\right\}
$$

For this let $z_{0}$ be a point in the set on the left-hand side. We choose an integer $l_{0}$ such that $\left|u_{l_{0}+j+1}\left(z_{0}\right)-u_{j}\left(z_{0}\right)\right| \geq \delta / 4$. Assume $z_{0} \notin \bigcup_{l=0}^{l_{0}-1}\left\{\left|u_{l+j+1}-u_{l+j}\right| \geq\right.$ $\left.\delta / 2^{l+j+2}\right\}$. Then

$$
\begin{aligned}
\left|u_{l_{0}+j+1}\left(z_{0}\right)-u_{l_{0}+j}\left(z_{0}\right)\right| & \geq\left|u_{l_{0}+j+1}\left(z_{0}\right)-u_{j}\left(z_{0}\right)\right|-\sum_{l=0}^{l_{0}-1}\left|u_{l+j+1}\left(z_{0}\right)-u_{l+j}\left(z_{0}\right)\right| \\
& \geq \frac{\delta}{4}-\sum_{l=0}^{l_{0}-1} \frac{\delta}{2^{l+j+2}} \geq \frac{\delta}{2^{l_{0}+j+2}},
\end{aligned}
$$

which implies $z_{0} \in\left\{\left|u_{l_{0}+j+1}-u_{l_{0}+j}\right| \geq \delta / 2^{l_{0}+j+2}\right\}$, and hence the above inclusion holds. So we have

$$
\begin{gathered}
C_{n}\left\{\left|\sup \left\{u_{j}, u_{j+1}, \ldots\right\}-u_{j}\right| \geq \frac{\delta}{2}\right\} \leq \sum_{l=0}^{\infty} C_{n}\left\{\left|u_{l+j+1}-u_{l+j}\right| \geq \frac{\delta}{2^{l+j+2}}\right\} \\
\leq \sum_{l=0}^{\infty} \frac{2^{n(l+j+2)}}{\delta^{n} 2^{(n+1)(l+j)}}=\frac{4^{n}}{\delta^{n} 2^{j}} \longrightarrow 0, \quad \text { as } j \rightarrow \infty .
\end{gathered}
$$

Therefore $u_{j} \rightarrow g$ in $C_{n}$-capacity on each compact subset $E$ of $\Omega$ and it then turns out from Theorem 1 that $\left(d d^{c} u_{j}\right)^{n} \rightarrow\left(d d^{c} g\right)^{n}$ as currents. Hence $\mu=\left(d d^{c} g\right)^{n}$ in $\Omega$ and the proof is complete.

As a consequence of Theorem 4 we also get the following result in [C-S].

Corollary. Suppose that $\Omega$ is a bounded domain in $\mathbb{C}^{n}$. If there exists a function $v \in P S H(\Omega) \cap L^{\infty}(\Omega)$ such that $\left(d d^{c} v\right)^{n} \geq f d \lambda$, where the function $f \in L^{1}(\Omega)$, then there exists a function $u \in P S H(\Omega) \cap L^{\infty}(\Omega)$ such that $\left(d d^{c} u\right)^{n}=f d \lambda$.

Proof. Since every non-negative integrable function is, in the $L^{1}$-norm, the limit of some sequence of non-negative continuous functions with compact support, then Corollary follows from Theorem D in [B-T1] and Theorem 4.

Note that without the assumption of the existence of a subsolution, neither Theorem 4 nor Corollary is true, as can be seen from the fact that there exists a positive measure $\mu=f d \lambda$ with $f \in L^{1}(\Omega)$ which is not the Monge-Ampère measure of a bounded psh function, see [C-S]. 


\section{REFERENCES}

[B-T1] E. Bedford and B. A. Taylor, The Dirichlet problem for the complex Monge-Ampère operator, Invent. Math. 37 (1976), 1-44. MR 56:3351

[B-T2] , A new capacity for plurisubharmonic functions, Acta Math. 149 (1982), 1-40. MR 84d:32024

[C] U. Cegrell, Discontinuité de l'opérateur de Monge-Ampère complexe, C. R. Acad. Sci. Paris Ser. I Math. 296 (1983), 869-871. MR 84j:32022

[C-S] U. Cegrell and A. Sadullaev, Approximation of plurisubharmonic functions and the Dirichlet problem for the complex Monge-Ampère operator, Math. Scand. 71 (1993), 62-68. MR 94d:32017

[L1] P. Lelong, Fonctions plurisousharmoniques et formes differentielles positives, Gordon and Breach, Paris, 1968. MR 39:4436

[L2] _ Discontinuité et annulation de l'opérateur de Monge-Ampère complexe, Lecture Notes in Math., vol. 1028, Springer-Verlag, Berlin, 1983, pp. 219-224. MR 86j:32038

[R] L. I. Ronkin, Weak convergence of the current $\left[d d^{c} u_{t}\right]^{q}$ and asymptotics of the finite order function for holomorphic mappings of regular growth, Sibirskiı̌ Mat. Zh. 25:4 (1984), 167173. MR 86f: 32027

[X] Y. Xing, On convergence of the current $\left(d d^{c} u_{t}\right)^{q}$, Séminaire P. Lelong-P. DolbeaultH. Skoda, Lecture Notes in Math. (to appear).

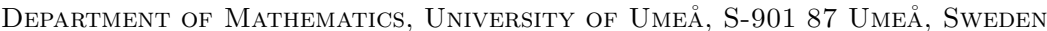

E-mail address: yang.xing@mathdept.umu.se 